\title{
A Characterization of 4-Centralizer Groups
}

\author{
Jutirekha Dutta \\ Department of Mathematics, North-Eastern Hill University, Permanent Campus, Shillong, Meghalaya 793022, India
}

Correspondence should be addressed to Jutirekha Dutta; jutirekhadutta@yahoo.com

Received 23 August 2013; Accepted 9 October 2013

Academic Editors: M. Coppens, W. Shi, and Z. Wang

Copyright (C) 2013 Jutirekha Dutta. This is an open access article distributed under the Creative Commons Attribution License, which permits unrestricted use, distribution, and reproduction in any medium, provided the original work is properly cited.

A finite or infinite group is called an $n$-centralizer group if it has $n$ numbers of distinct centralizers. In this paper, we prove that a finite or infinite group $G$ is a 4 -centralizer group if and only if $G / Z(G)$ is isomorphic to $C_{2} \times C_{2}$. This extends a result of Belcastro and Sherman.

\section{Introduction}

Given a finite or infinite group $G$ and $x \in G$, the set $C(x)=$ $\{y \in G \mid x y=y x\}$ is called the centralizer of $x$ in $G$. The set of all centralizers in $G$ is denoted by $\operatorname{Cent}(G)$. A group $G$ is called an $n$-centralizer group if $|\operatorname{Cent}(G)|=n$. It is easy to see that one-centralizer groups are precisely the abelian groups. Characterization of finite groups in terms of the number of distinct centralizers has been an interesting topic of research in recent years (see [1-8]). In [7], Belcastro and Sherman have proved the nonexistence of finite $n$-centralizer group for $n=2,3$. However, their proof also shows the nonexistence of infinite 2,3-centralizer groups. Belcastro and Sherman [7] also characterize all finite 4-centralizer groups. More precisely, they proved that a finite group $G$ is a 4 centralizer group if and only if $G / Z(G)$ is isomorphic to $C_{2} \times C_{2}$, where $Z(G)$ is the center of $G$ and $C_{2}$ is the cyclic group having two elements. In this paper, we extend the same characterization for infinite groups using elementary techniques of group theory.

Throughout this paper $G$ will denote a finite or infinite group. Recall that for any group $G$, its center $Z(G)$ is the intersection of all centralizers in $G$. Also $G$ is the union of all the centralizers of noncentral elements of $G$. It may be mentioned here that a finite or infinite group can not be written as union of two of its proper subgroups. These facts have important role in proving the main theorem of this paper.

\section{Main Result}

In this section, we proof the following main theorem of this paper.

Theorem 1. A finite or infinite group $G$ is a 4-centralizer group if and only if $G / Z(G) \cong C_{2} \times C_{2}$.

Proof. Let $G$ be a 4-centralizer finite or infinite group and $\operatorname{Cent}(G)=\{G, C(p), C(q), C(r)\}$, where $p, q$, and $r$ are noncentral elements of $G$. Then $G=C(p) \cup C(q) \cup C(r)$, since $G$ is the union of its proper centralizers.

Let us consider the centralizer $C(p q)$. Then $C(p q)$ will be one of $G, C(p), C(q)$, or $C(r)$.

If $C(p q)=G$, then $p q \in Z(G)$. This implies that $p=$ $z q^{-1}$ for some $z \in Z(G)$. Therefore, we get $C(p)=C(q)$, a contradiction. If $C(p q)=C(p)$, then $g \in C(p)=C(p q)$ gives

$$
g p q=p q g \Longrightarrow p g q=p q g \Longrightarrow g \in C(q) \text {. }
$$

Therefore, $C(p) \subseteq C(q)$. Hence, $G=C(q) \cup C(r)$, a contradiction, as a group can not be written as union of two of its proper subgroups. Similarly, it can be seen that $C(p q) \neq C(q)$.

Thus, $C(p q)=C(r)$ and so $G=C(p) \cup C(q) \cup C(p q)$. In a similar way it can be seen that $C(q p)=C(r)$ and so $C(p q)=$ $C(q p)$. 
We will now show that $C(p) \cap C(q)=Z(G)$. Clearly, $Z(G) \subseteq C(p) \cap C(q)$. Let $g \in C(p) \cap C(q)$. Then

$$
\begin{aligned}
g p & =p g \Longrightarrow(g p) q=(p g) q \Longrightarrow g p q \\
& =p q g \Longrightarrow g \in C(p q) .
\end{aligned}
$$

Therefore, $g \in C(p) \cap C(q) \cap C(p q)=Z(G)$ and so $C(p) \cap$ $C(q) \subseteq Z(G)$. Thus,

$$
C(p) \cap C(q)=Z(G) .
$$

In a similar way, it can be seen that $C(q) \cap C(p q)=Z(G)$, and $C(p) \cap C(p q)=Z(G)$.

Let us consider the right cosets $Z, Z p, Z q$, and $Z p q$, where $Z:=Z(G)$. As $p$ is a noncentral element of $G$ it follows that $Z \neq Z p, Z q$, and $Z p q$.

If $Z p=Z q$, then we have $p=z q$ for some $z \in Z$. Therefore, we get $C(p)=C(q)$, a contradiction. Again, if $Z p=Z p q$, then $(p q)^{-1} p \in Z$ which gives $q \in Z$, a contradiction. Similarly, it can be seen that $Z q \neq Z p q$. Thus the cosets $Z, Z p, Z q$, and $Z p q$ are mutually disjoint. Clearly, $Z \cup Z p \cup Z q \cup Z p q \subseteq G$.

Let $g \in Z$ then $g \in Z \cup Z p \cup Z q \cup Z p q$. Suppose that $g \in G-Z$ then without any loss of generality we may assume that $g \in C(p)$. Let us consider the centralizer $C(g q)$. Then $C(g q)$ is one of $G, C(p), C(q)$, or $C(p q)$.

Case 1. Let $C(g q)=G$. In this case, $g q \in Z(G)$ and so $g q q=$ $q g q$ which gives $g \in C(q)$. Therefore, $g \in C(p) \cap C(q)=$ $Z(G)$, a contradiction.

Case 2. Let $C(g q)=C(p)$. In this case, $g q p=p g q$ which gives $p \in C(q)$. Therefore, $p \in C(p) \cap C(q)=Z(G)$, a contradiction.

Case 3. Let $C(g q)=C(q)$. In this case, $g q q=q g q$ which gives $g \in C(q)$. Therefore, $g \in C(p) \cap C(q)=Z(G)$, a contradiction.

Hence, $C(g q)=C(p q)$, since $G$ is a 4-centralizer group. Now, $C(g q)=C(p q)$ implies that $g q p=p q g$ which gives $g p^{-1} q=q g p^{-1}$ since $g \in C(p)$. Thus, $g p^{-1} \in C(q)$. Also, $g \in C(p)$ implies that $g p^{-1} \in C(p)$. Hence, $g p^{-1} \in C(p) \cap$ $C(q)=Z(G)$ and so $g=z p$ for some $z \in Z(G)$. In other words, $g \in Z p$. Therefore, $g \in Z \cup Z p \cup Z q \cup Z p q$. Hence, $G=Z \cup Z p \cup Z q \cup Z p q$. This shows that $|G / Z(G)|=4$. Finally, the fact that $G$ is nonabelian gives $G / Z(G) \cong C_{2} \times C_{2}$.

Conversely, let $G / Z(G) \cong C_{2} \times C_{2}$. Therefore, there are four right cosets of $Z=Z(G)$ in $G$, namely, $Z, Z p, Z q$, and $Z r$, where $p, q$, and $r$ are distinct noncentral elements of $G$. So, for any element $g \in G$, either $g \in Z$ or $Z p$ or $Z q$ or $Z r$. If $g \in Z$, then $C(g)=G$. If $g \in Z p$, then $g=z p$ for some $z \in Z$, therefore $C(g)=C(z p)=C(p)$. Similarly, $g \in Z q$ gives $C(g)=C(q)$ and $g \in Z r$ gives $C(g)=C(r)$. Hence, $G$ has at most four centralizers, namely, $G, C(p), C(q)$, and $C(r)$. Since $|\operatorname{Cent}(G)| \geq 4$ we have $|\operatorname{Cent}(G)|=4$. This completes the proof.

We conclude this paper by the following remark. In [7], Belcastro and Sherman proved Theorem 1 for finite groups only. Note that their proof can not be extended to infinite groups as they have used the finiteness of the group extensively. Belcastro and Sherman [7] have also obtained the structure of 5-centralizer finite groups as follows.

A finite group $G$ is a 5-centralizer group if and only if $G / Z(G) \cong C_{3} \times C_{3}$ or $G / Z(G) \cong S_{3}$, where $C_{3}$ is the cyclic group having three elements and $S_{3}$ the symmetric group on three symbols.

Note that the if part of the above result also holds for infinite groups. The same proof of Belcastro and Sherman [7] holds for infinite groups. However, the proof of the only if part of the above result is not known for infinite groups. It may be interesting to study the infinite $n$-centralizer groups for $n>4$.

\section{Acknowledgments}

This paper is a part of the author's M. Phil. thesis done under the supervision of Professor A. K. Das. The author would like to thank him for his helpful suggestions. The author is grateful to all the referees for their valuable comments and suggestions.

\section{References}

[1] A. Abdollahi, S. M. J. Amiri, and A. M. Hassanabadi, "Groups with specific number of centralizers," Houston Journal of Mathematics, vol. 33, no. 1, pp. 43-57, 2007.

[2] A. R. Ashrafi, "On finite groups with a given number of centralizers," Algebra Colloquium, vol. 7, no. 2, pp. 139-146, 2000.

[3] A. R. Ashrafi, "Counting the centralizers of some finite groups," The Korean Journal of Computational \& Applied Mathematics, vol. 7, no. 1, pp. 115-124, 2000.

[4] A. R. Ashrafi and B. Taeri, "On finite groups with a certain number of centralizers," Journal of Applied Mathematics and Computing, vol. 17, no. 1-2, pp. 217-227, 2005.

[5] A. R. Ashrafi and B. Taeri, "On finite groups with exactly seven element centralizers," Journal of Applied Mathematics and Computing, vol. 22, no. 1-2, pp. 403-410, 2006.

[6] S. J. Baishya, "On finite groups with specific number of centralizers," International Electronic Journal of Algebra, vol. 13, pp. 5362, 2013.

[7] S. M. Belcastro and G. J. Sherman, "Counting centralizers in finite groups," Mathematics Magazine, vol. 67, no. 5, pp. 366374, 1994.

[8] M. Zarrin, “On element-centralizers in finite groups," Archiv der Mathematik, vol. 93, no. 6, pp. 497-503, 2009. 


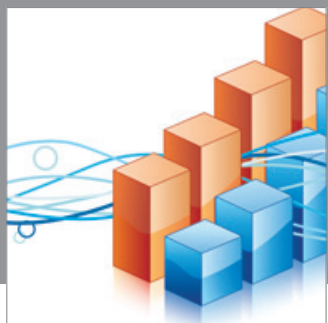

Advances in

Operations Research

mansans

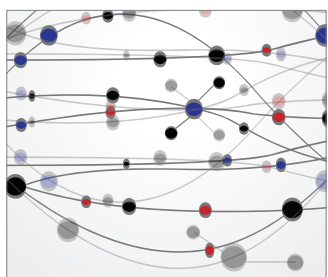

The Scientific World Journal
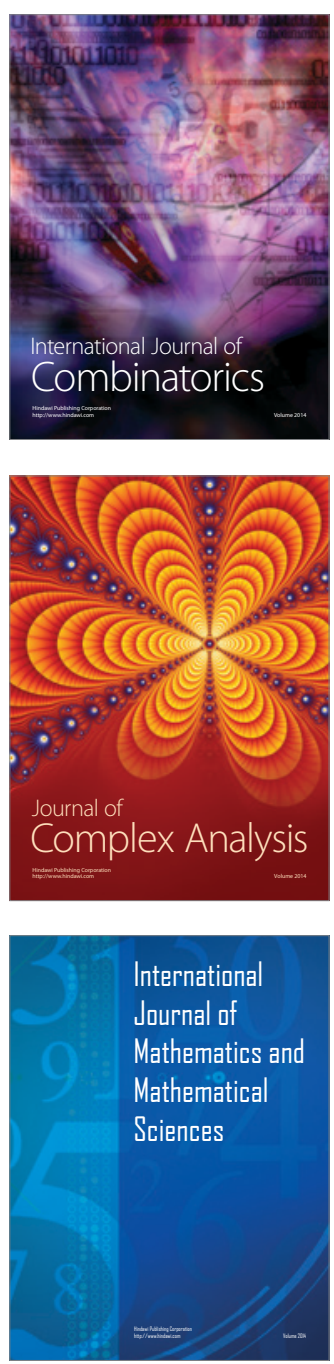
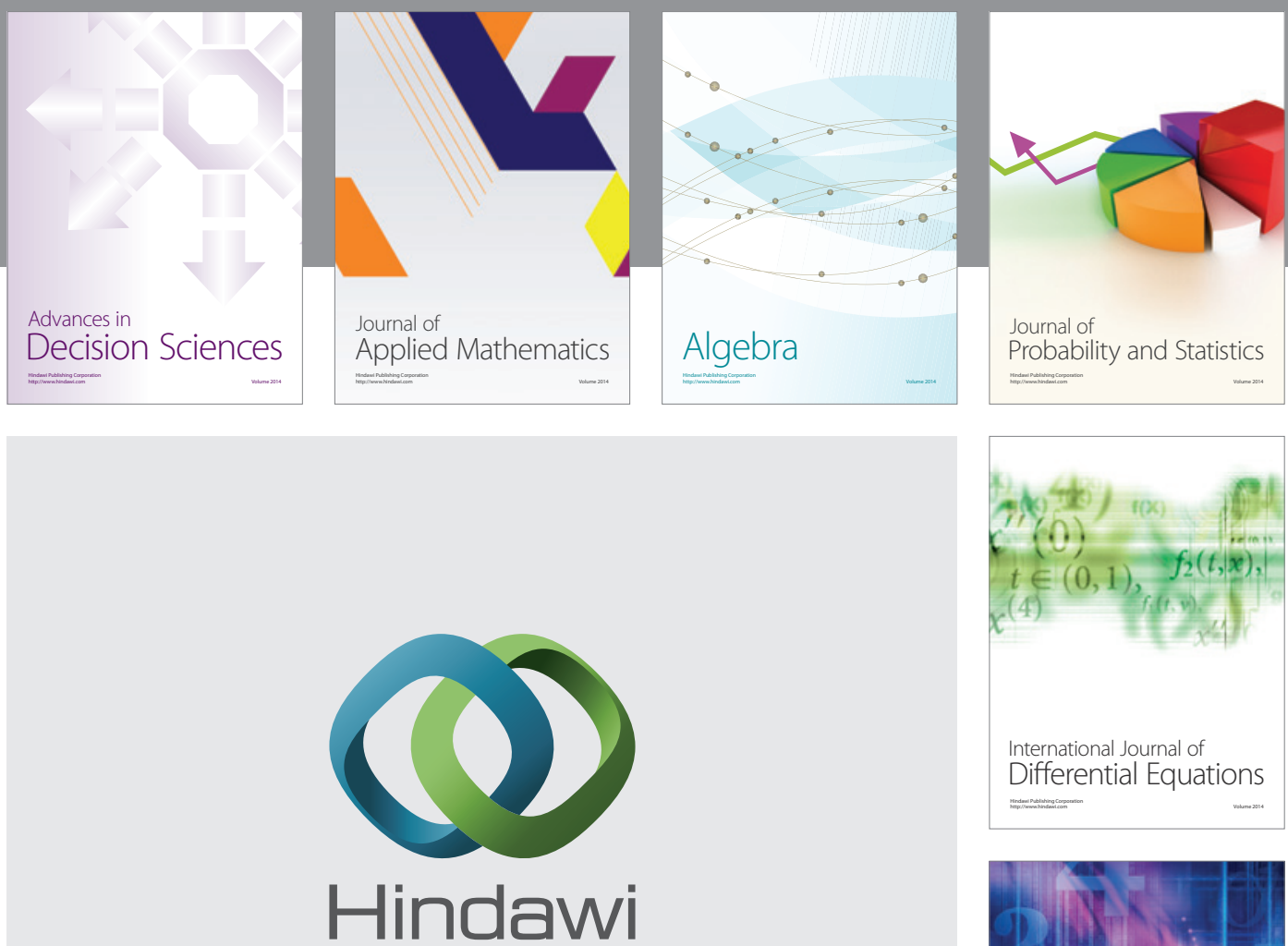

Submit your manuscripts at http://www.hindawi.com
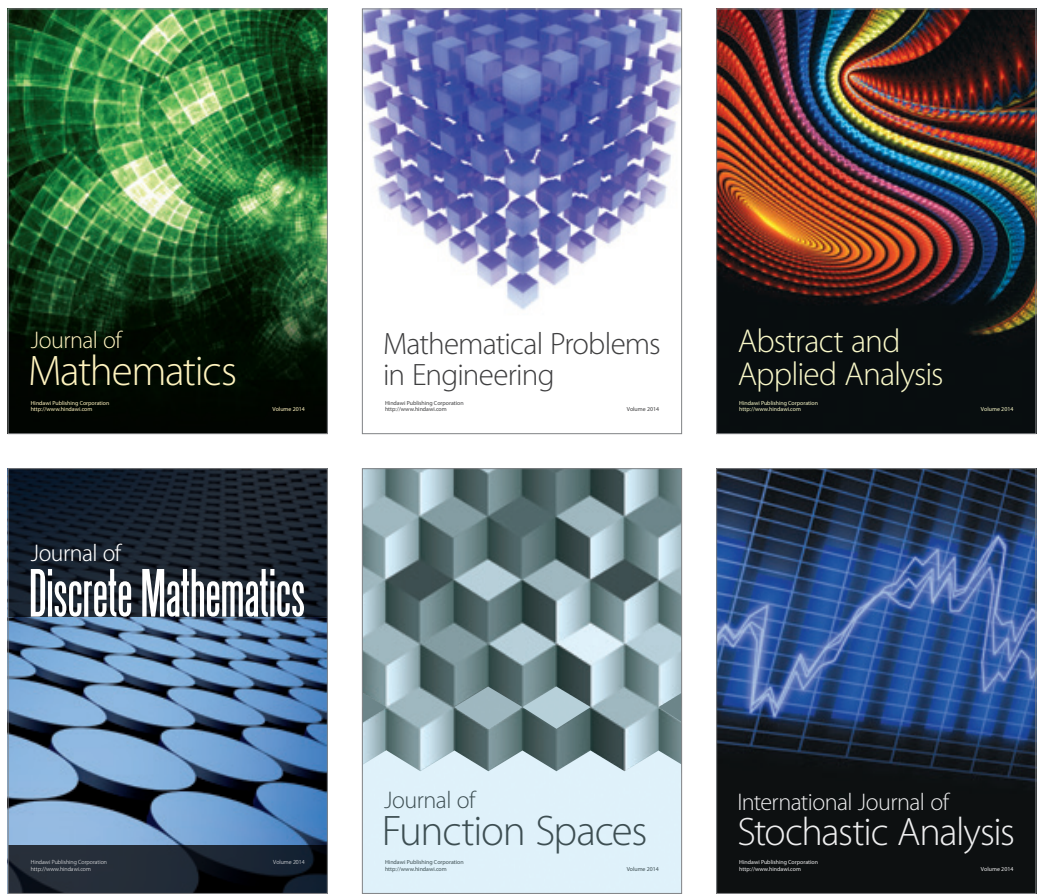

Journal of

Function Spaces

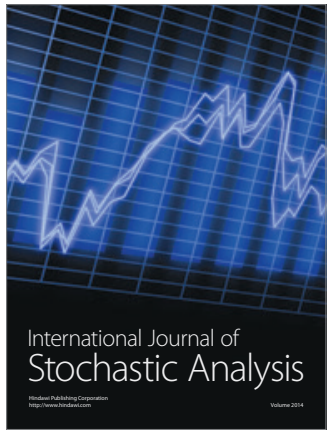

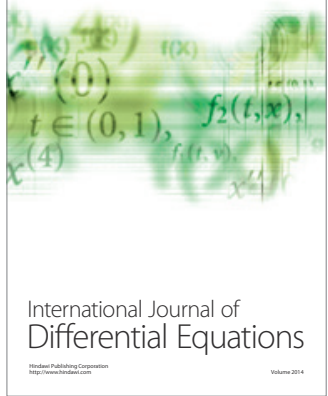
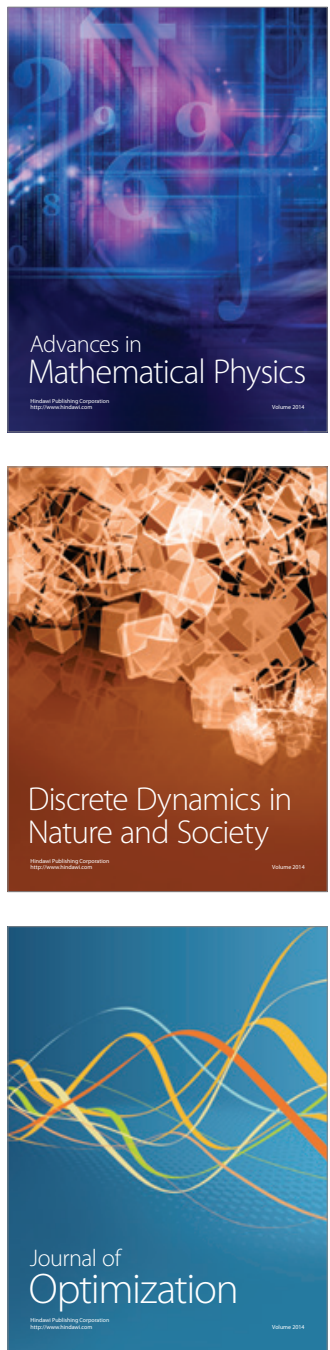